\title{
Do the $\gamma$ Cas X-rays come from the Be Star?
}

\author{
Myron A. Smith ${ }^{1}$ and R. Lopes de Oliveira ${ }^{2}$ \\ ${ }^{1}$ Dept. of Physics \& Astronomy, Catholic University of America, \\ Washington, D.C. 20064, USA \\ email: msmith@stsci.edu \\ ${ }^{2}$ Universidade de São Paulo, Instituto de Física de São Carlos, \\ Caixa Postal 369, 13560-970, São Carlos, SP, Brazil
}

\begin{abstract}
We discuss the origin of the hard X-rays in $\gamma$ Cas and its analogs. Of great importance are their temporal correlations with optical/UV signatures, suggesting an origin near the Be star.
\end{abstract}

Keywords. stars: emission-line, Be, stars: winds, outflows, stars: magnetic fields, X-rays: stars

\section{Introduction}

$\gamma$ Cas (B0.5IVe) is the prototype of a group of some 8 known Galactic B0.5-1e stars with hot thermal $(k T \geqslant 10 \mathrm{keV}) \mathrm{X}$-ray spectra indicating a complex geometry and strong $\mathrm{H} \alpha$ emission. $\gamma$ Cas itself exhibits continuous flaring on timescales of a few seconds (requiring densities of $\rho>10^{14} \mathrm{~cm}^{-3}$; see "scatter" in Fig. 1), a few hours, and 2-3 month cycles. The origin of the X-rays is a mystery. Multiband correlations suggest that they could arise from physical interactions near the Be star, e.g. from magnetic stresses arising from differential rotation between solid-body rotation near the surface and the Keplerian disk.

\section{Lines of observational evidence}

Simultaneous X-ray/UV spectral campaign in January 1996 established strong correlations between flux variations of X-rays, the inverted UV continuum and in lines formed above the Be star from species below and above the dominant ion stage(see Fig. 1). X-ray flux seems to influence the ionization of the local circumstellar plasma.

At times of high X-ray flux, light dips are too brief to be due to surface spots and must be due to passages of translucent clouds $\sim 0.3 \mathrm{R}_{*}$ above the surface (Smith et al. 1998), consisting of both warm and cool plasma (Smith \& Robinson 1999). Spectral lines in the optical and UV spectrum of $\gamma$ Cas frequently exhibit "migrating subfeatures" in absorption. These msf move blue to red across the profile with an acceleration of $+95 \mathrm{~km} \mathrm{~s}^{-2}$ and arise in small cloudlets in front of the star at mainly the same times as the UV flux dips. The msf are also present in optical lines of the $\gamma$ Cas analog star HD 110432 (Smith \& Balona 2006) and AB Dor, which is a well known active K dwarf with a complex magnetic field configuration. All of these activities find a common explanation in CS clouds forced into corotation near X-ray active centers on the Be star. This inference is supported by the finding of overbroadening in the weakest hydrogen Brackett emission lines of the $\gamma$ Cas spectrum, indicating that the inner CS region rotates more rapidly than the Be star (Hony et al. 2000), where vsin $i \approx 400 \mathrm{~km} \mathrm{~s}^{-1}$.

The light curve of $\gamma$ Cas shows periodic, gray nonsinusoidal signatures of amplitude 0.003 mags. that are stable over 10 years. The period is $1.21581 \pm 0.00004$ days (Smith et al. 2006). This is undoubtedly a signature of rooted surface chemical inhomogeneities. There are no periodic UV resonance line variations. Thus the inferred presence of surface fields must take the form of a complex topology. 
(a)

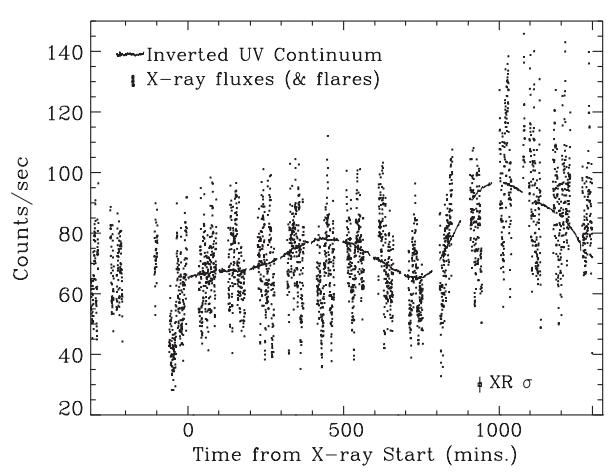

(b)

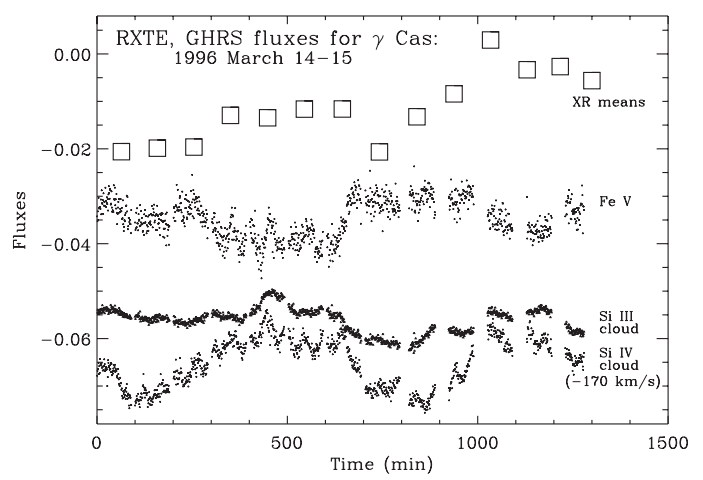

Figure 1. (a) X-ray and inverted UV continuum fluxes; (b) X-ray with Si III, Si IV and Fe V line flux.

The same optical light curve shows 2-3 month-long optical cycles with larger amplitudes in the red. In 2000 and 2001 these cycles were found to be well correlated with X-ray cycles (Robinson et al. 2002). (At different times a similar X-ray and optical cycle has been found in the $\gamma$ Cas analog HD 110432.) Robinson \& Smith (2000) suggested that variable physical conditions in the disk, e.g. a magnetic dynamo, mediate the X-ray emission.

Our 1996 HST/GHRS spectra disclose the presence of high velocity absorptions, up to $+2000 \mathrm{~km} \mathrm{~s}^{-1}$ (Smith \& Robinson 1999), implying that CS blobs accelerate toward the star. These velocities are sufficient to excite the X-ray flares observed in $\gamma$ Cas.

Putting this all together, we believe correlations between X-ray and UV/optical diagnostics show the X-rays are produced near or on the surface of the Be star. We speculate that shear in angular rotation between the solid-body CS complexes near the Be star and its Keplerian disk cause magnetic lines of force to stretch, break, and reconnect, thereby catapulting ambient debris towards the star and causing copious hard X-ray emission.

The building blocks for this idea to work are a rapidly rotating Be star, possibly a complex surface field, and a robust disk. $\gamma$ Cas itself is a 204 day binary in a nearly circular orbit (Miroshnichenko et al. 2002). This condition and a case for two other $\gamma$ Cas analogs being blue stragglers suggest the possibility that prior prior angular momentum could have spun up the Be star. When occurring together these conditions would satisfy the requirement that the $\gamma$ Cas stars occur infrequently but are not unique.

In our view these observations seem to point to Be star-disk interactions. Yet, it cannot be corroborated to date that any specific mechanism that mediates X-ray variability, such as a magnetorotational instability, is self-sustaining or has predictive power.

\section{References}

Hony, S., Waters, L. B. F. M., Zaal, P. A., \& de Koter, A. et al. 2000, A\&̊A, 355, 187

Miroshnichenko, A. S., Bjorkman, K. S., \& Krugov, V. D. 2002, PASP, 114, 1226

Robinson, R. D., Smith, M. A., \& Henry, G. W. 2002, ApJ, 575, 435

Robinson, R. D. \& Smith, M. A. 2000, ApJ, 540, 474

Smith, M. A., Robinson, R. D. \& Hatzes, A. P. 1998, ApJ, 507, 945

Smith, M. A. \& Balona, L. 2006, ApJ, 640, 491

Smith, M. A. \& Robinson, R. D. 1999, ApJ, 517, 866

Smith, M. A., Henry, G. W., \& Vishniac, E. 2006, ApJ, 647, 1375 